\title{
Characteristics, Management, and Case-Fatality of Patients Hospitalized for Stroke with a Diagnosis of COVID-19 in France
}

\author{
Amélie Gabet $^{\mathrm{a}}$ Clémence Grave ${ }^{\mathrm{a}}$ Edouard Chatignoux ${ }^{\mathrm{a}} \quad$ Philippe Tuppin $^{\mathrm{b}}$ \\ Yannick Béjot ${ }^{c}$ Valérie Olié ${ }^{a}$ \\ aDepartment of Non-Communicable Diseases, Cardiovascular Diseases and Stroke Program, Santé Publique France, \\ Saint-Maurice, France; 'bepartment of strategy and statistical studies, Caisse Nationale d'Assurance Maladie, Paris, \\ France; 'Neurology Department, Dijon University Hospital, Dijon, France
}

\section{Keywords}

Stroke $\cdot$ COVID-19 Epidemiology $\cdot$ Stroke management .

Mortality

\begin{abstract}
Introduction: COVID-19 was found to be associated with an increased risk of stroke. This study aimed to compare characteristics, management, and outcomes of hospitalized stroke patients with or without a hospital diagnosis of COVID-19 at a nationwide scale. Methods: This is a cross-sectional study on all French hospitals covering the entire French population using the French national hospital discharge databases (Programme de Médicalisation des Systèmes d'Information, included in the Système National des Données de Santé). All patients hospitalized for stroke between 1 January and 14 June 2020 in France were selected. A diagnosis of COVID-19 was searched for during the index hospitalization for stroke or in a prior hospitalization that had occurred after 1 January 2020. Results: Among the 56,195 patients hospitalized for stroke, $800(1.4 \%)$ had a concomitant COVID-19 diagnosis. Inhospital case-fatality rates were higher in stroke patients with COVID-19, particularly for patients with a primary diagnosis of COVID-19 (33.2\%), as
\end{abstract}

karger@karger.com

(c) 2021 S. Karger AG, Basel

www.karger.com/ned

Karger" compared to patients hospitalized for stroke without COVID-19 diagnosis (14.1\%). Similar findings were observed for 3 -month case-fatality rates adjusted for age and sex that reached $41.7 \%$ in patients hospitalized for stroke with a concomitant primary diagnosis of COVID-19 versus $20.0 \%$ in strokes without COVID-19. Conclusion: Patients hospitalized for stroke with a concomitant COVID-19 diagnosis had a higher inhospital and 3 months case-fatality rates compared to patients hospitalized for stroke without a COVID-19 diagnosis. Further research is needed to better understand the excess of mortality related to these cases.

(c) 2021 S. Karger AG, Basel

\section{Introduction}

As other respiratory tract infections, severe acute respiratory syndrome-coronavirus-2 (SARS-CoV-2) infection is associated with a higher risk of stroke, particularly ischemic stroke [1]. Several studies described stroke patients infected with SARS-CoV-2 [1-3]. However, very few data on large population size were published and none on an exhaustive national population at the exception of Germany [4]. Furthermore, previous studies 
mainly compared characteristics and outcomes of COVID-19 patients developing stroke to COVID-19 patients free of stroke [1]. The aim of this cross-sectional nationwide study was to describe characteristics, acute care management, and outcomes for patients hospitalized for stroke with a diagnosis of COVID-19 in comparison to patients hospitalized for stroke without simultaneous COVID-19 diagnosis.

\section{Methods}

\section{Population Selection}

Patients hospitalized for stroke in the whole French territory between the 1 January 2020 and 14 June 2020 were selected using the French national hospital discharge databases (Programme de Médicalisation des Systèmes d'Information, included in the Système National des Données de Santé) which exhaustively registered all hospital stays from both private and public sectors in France. Stroke was defined using the codes of international classification of diseases - 10th revision (ICD-10) I60-I64, considered as the main diagnosis of the entire hospital stay or for one of the medical units where the patient stayed. Hospital diagnosis of COVID-19 was based on the identification of SARS-CoV-2 through a biologic test (PCR, antigen, or serologic), or based on a CT scan. The first cases of COVID-19 imported in France were confirmed on 24 January 2020 [5]. As COVID-19 incidence started to increase exponentially in February [5], only few cases of COVID-19 would be observed among patients hospitalized for stroke in January and February.

Among hospitalized stroke patients with a COVID-19 diagnosis, 3 groups were distinguished according to the position of the COVID-19 diagnosis: reported in a prior hospitalization before the index hospitalization for stroke (group A), registered as the main diagnosis of the entire hospital stay for stroke or one of the medical units where the patient stayed (group B), and registered as an associated diagnosis of the index hospitalization for stroke (group C). For patients of the group A, infection to SARS-CoV-2 preceded the stroke, whereas no temporal assertion between stroke and COVID-19 could be made for patients from groups B or C. Finally, the distinction between groups $\mathrm{B}$ and $\mathrm{C}$ allowed to clarify the intensity of COVID-19. Indeed, COVID-19 motivated the hospitalization or required acute specific care for the group $\mathrm{B}$, contrary to the group $\mathrm{C}$ for which COVID-19 was registered as an associated diagnosis only. Furthermore, a COVID-19 code recorded as a main diagnosis meant higher severity related to COVID-19 than a COVID-19 code registered as an associated diagnosis. Finally, we compared overall patients hospitalized for stroke with a COVID-19 diagnosis (groups A, B, and C altogether) to those without COVID-19.

\section{Data Collected}

Age, sex, type of stroke (ischemic [ICD-10 codes I63 and I64], undetermined stroke was ischemic strokes from undetermined etiology), hemorrhagic (including subarachnoid hemorrhage [ICD10 codes I60], intracerebral hemorrhage [ICD-10 codes I61], and other or unprecised hemorrhage [ICD-10 codes I62], and patients with both ischemic and hemorrhagic stroke which included hem- orrhagic transformation of ischemic strokes), a history (hospitalization or long-term disease status for a disease that entitles $100 \%$ reimbursement for all healthcare expenditures related to that particular disease) of several cardiovascular diseases recorded in the 5 previous years, and drug treatments with at least 3 deliveries in the 12 months preceding the index hospitalization for stroke were recorded. Inhospital mortality was recorded for all patients. Mortality at 3 months after admission to hospital for stroke was recorded for patients without error linkage between the hospital discharge databases and the databases including vital status $(N=54,534)$, and affiliated to the general insurance scheme $(N=40,988)$, that is, $75.2 \%$ of patients hospitalized for stroke in our study. An additional 10 patients were excluded due to wrong date of death leading to a population of 40,978 patients for whom mortality at 3 months after the admission at hospital for stroke was available.

\section{Statistical Analysis}

Characteristics of patients hospitalized for stroke were compared between those with a COVID-19 diagnosis and those without a COVID-19 diagnosis using the $\chi^{2}$ test and Fisher's test when necessary for categorical variables, and using the Wilcoxon-MannWhitney test for quantitative variables. Means and standard deviation were calculated for each quantitative characteristic. Analyses by specific type of stroke, that is, ischemic and hemorrhagic, were conducted and presented in online suppl. material; see www. karger.com/doi/10.1159/000516670 for all online suppl. material, for patients characteristics and resumed in Table 2 for outcomes. Analyses were performed using SAS Enterprise Guide 7.1.

\section{Data Availability}

Full access to the Programme de Médicalisation des Systèmes d'Information and the Système National des Données de Santé is granted to Santé Publique France by decree.

\section{Results}

Of the 56,195 patients hospitalized for stroke in France between January and the middle of June 2020, 800 patients (1.4\%) had a diagnosis of COVID-19 reported on their hospital medical file, including 157 patients having a COVID-19 diagnosis already mentioned in a prior hospitalization (group A), 211 patients with a primary diagnosis of COVID-19 (group B), and 432 patients with COVID-19 recorded as an associated diagnosis only (group C). The median delay between a prior hospitalization with a COVID-19 diagnosis and the hospitalization for stroke (group A only) was 19 days (IQR: 6.5-39.5). Similar proportions of COVID-19 diagnosis were found among ischemic (1.4\%) and hemorrhagic (1.5\%) strokes (see online suppl. Tables 1,2). The diagnosis of COVID-19 was based on a biologic test in $68.0 \%$ of cases versus on a CT scan in $32.0 \%$ of stroke cases (Table 1 ).

Stroke patients with COVID-19 had a similar age and proportion of women as compared to stroke patients 


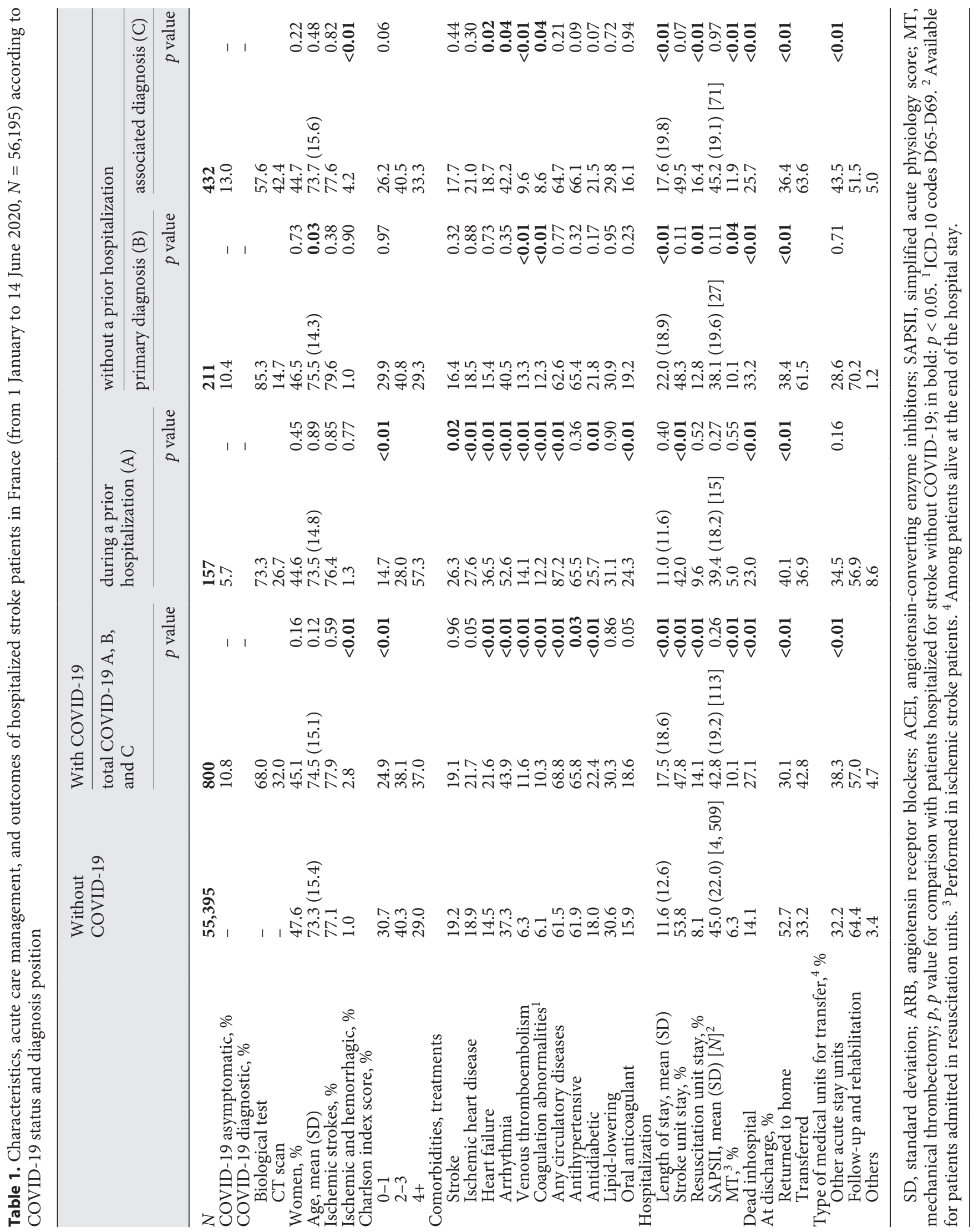




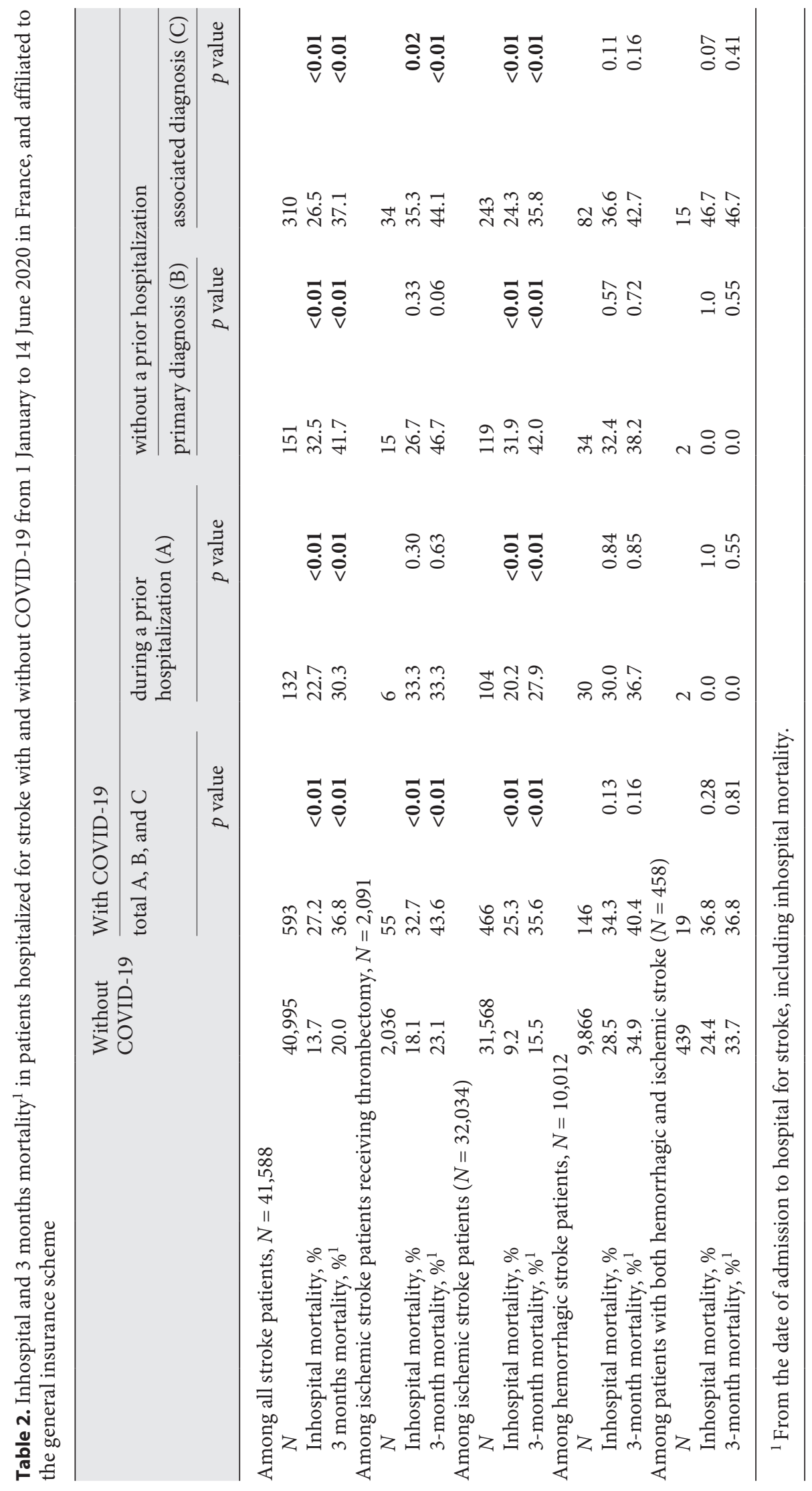


without COVID-19 (Table 1), and whatever the type of stroke (see online suppl. Tables 1, 2). However, ischemic strokes with a COVID-19 diagnosis receiving mechanical thrombectomy (MT) were younger than those receiving MT without a COVID-19 diagnosis $(66.9[n=55]$ vs. 70.9 $[n=2,036], p=0.04$, data not shown) and were more frequently men ( 66 vs. $48 \%, p=0.02$, data not shown). Patients with a COVID-19 diagnosis recorded on a prior hospitalization (group A) had different characteristics than stroke patients without COVID-19, with a higher number of comorbidities $(4 \leq$ Charlson index score for 57.3 vs. $29.0 \%$ and Charlson index score of $0-1$ for 14.7 vs. $30.7 \%, p<0.01$ ) (Table 1 ). This observation was found among both ischemic and hemorrhagic strokes (see online suppl. Tables 1,2 respectively).

Patients hospitalized for stroke with COVID-19 were more severe cases than patients hospitalized for stroke without COVID-19 as shown by a higher proportion of MT (10.1 and 11.9\% for groups B and C, respectively vs. $6.3 \%$ in non-COVID-19), and a higher inhospital casefatality rate $(23.0,33.2$, and $25.7 \%$ for groups $A, B$, and C, respectively vs. $14.1 \%$ in non-COVID-19) (Table 2). Three months case-fatality remained higher in groups $\mathrm{A}$ (30.3\%), B (41.7\%), or C (37.1\%) as compared to patients hospitalized for stroke without COVID-19 (20.0\%), and in both ischemic and hemorrhagic strokes (Table 2).

\section{Discussion}

This study was one of the largest reporting inhospital outcomes of patients hospitalized for stroke with a prior or concomitant COVID-19 diagnosis at a nationwide scale. These patients seemed to have more severe strokes as compared to patients without a COVID-19 diagnosis regarding inhospital and 3-month case-fatality rates. Nevertheless, differences in the level of comorbidities, the mean length of stay, admission to resuscitation units, MT, and even inhospital mortality were observed according to the position of the COVID-19 diagnosis that reflected different intensity of COVID-19 disease.

The greater severity reflected by higher case-fatality rates is consistent with a meta-analysis which showed a higher NIHSS score in stroke patients with COVID-19 than in those without COVID-19, and higher inhospital case-fatality in presence of SARS-CoV-2 infection [6]. Furthermore, acute ischemic strokes due to large vessel occlusion were more common in COVID-19 stroke patients enrolled in this meta-analysis which is in line with the twice higher rate of MT in patients hospitalized for stroke with COVID-19 than those without COVID-19 in our study.

In our study, only stroke patients with COVID-19 registered before stroke (group A) had much higher prevalence of comorbidities (cardiovascular and noncardiovascular) as compared to stroke cases without COVID-19. This finding supports the hypothesis of a higher risk of developing severe COVID-19 in populations with greater proportion of comorbidities [7]. We observed twice higher inhospital and 3-month mortality in patients hospitalized for stroke with a primary diagnosis of COVID-19 (group B). This result might highlight the difficulty to manage patients with both acute stroke and severe COVID-19 in terms of care pathway organization including prehospital management, emergency department regulation, interhospital transfers, and endovascular procedures as found in previous studies $[8,9]$ from France [10], the stroke care network of Madrid [11], in Germany [4], in Lombardy and Italy [12]. These difficulties led to the publication of a guidance statement based on shared best practices in May 2020 [13]. Patients hospitalized for stroke with an associated diagnosis of COVID-19 (group C) had also a higher inhospital case-fatality rate than patients hospitalized for stroke without COVID-19, although they had lower inhospital case-fatality rates than patients hospitalized for stroke with a primary diagnosis of COVID-19 (group B). This suggested the higher severity of COVID-19-associated stroke found in previous studies $[2,14,15]$. However, we lacked clinical data to assess properly stroke severity. According to a review, COVID-19-related stroke patients showed higher prevalence of large vessel occlusion infarction and higher prevalence of atypical location of intracerebral hemorrhage [16].

No significant difference in the mean age or sex distribution between patients hospitalized for stroke with a COVID-19 diagnosis and those without a COVID-19 diagnosis was found in our study. Our results differed from the meta-analysis of 11 previous studies which showed that stroke patients with COVID-19 were significantly younger and were less likely to be women as compared to other stroke patients [1]. This meta-analysis reported no significant difference in hypertension, diabetes, dyslipidemia, or atrial fibrillation. To the opposite, we found different prevalence of these conditions in our study. Nevertheless, we approached vascular risk factors using dedicated medication use in the year preceding the index hospitalization for stroke, which could explain the discrepancies with the findings of Nannoni et al. [1]. 


\section{Strength and Limitations}

This study reported characteristics of stroke patients with prior or effective SARS-CoV-2 infection in one of the largest population sizes ever described. Although comparisons of characteristics between hospitalized stroke patients with COVID-19 and those without COVID-19 were described, interpretation of the results is limited regarding the unknown temporality of stroke and SARS-CoV-2 infection. Causality between SARS-CoV-2 infection and severity of stroke cannot be indeed concluded with our study design and our data, as we had access to hospitalization data only and not out of hospital COVID-19 diagnosis. Furthermore, a bias of classification might exist in our study regarding the COVID-19 diagnosis: we cannot exclude that some COVID-19 patients could remain in the non-COVID-19 group due to neither a biological test nor a chest-CT performed. This limitation could particularly impact the first weeks of the pandemic where a biological test had not already been widely used. In our study, most of COVID-19 diagnosis among stroke cases were based on a biologic test. According to a recent study by the stroke care network from Madrid, stroke patients with a diagnosis of COVID-19 confirmed by a biologic test had similar profile and outcomes than stroke patients diagnosed with a COVID-19 through a chest CT [11].

Associations between patients' characteristics and outcomes were not studied as some confounding factors were not exhaustively available, such as obesity, a known factor associated with increased severity in patients with COVID-19 [17]. Regarding stroke management, intravenous thrombolysis could not be exhaustively reported and was therefore not studied. No data about time to groin, other procedural data, or clinical characteristics such as the NIHSS score were available in French national hospital discharge databases. However, other comorbidities could be exhaustively recorded including diabetes and hypertension when treated. Finally, we were not able to identify the mechanism leading to ischemic stroke with the data use for this study, limiting the characterization of strokes with a concomitant COVID-19 diagnosis, or the mechanism leading to death limiting the interpretation of associated mortality.

\section{Conclusions}

Very poor outcomes were observed in patients hospitalized for stroke with a COVID-19 diagnosis as compared to patients hospitalized for stroke without CO-
VID-19. Further researches are needed to understand the pathophysiology of COVID-19-associated stroke in order to adjust acute care management.

\section{Statement of Ethics}

In line with the French governmental regulations and the National Ethics Committee, no patient consent was required. The databases used in the study contained pseudonymized patient information.

\section{Conflict of Interest Statement}

Yannick Béjot reports personal fees from AstraZeneca, BMS, Pfizer, Medtronic, MSD France, Amgen, Servier, and BoehringerIngelheim. None of the authors declared potential conflict of interest related to the submitted work.

\section{Funding Sources}

The authors did not receive any funding.

\section{Authors Contributions}

A.G. directed research, wrote the manuscript, and performed the statistical analyses; C.G. and E.C. contributed to statistical analyses, discussion, and reviewed the manuscript; P.T., Y.B., and V.O. directed research, contributed to discussion, and reviewed the manuscript. All the authors have read and approved the manuscript and its publication in Annals of Emergency Medicine as a Brief Research Report. Moreover, the authors declare no potential conflict of interest in relation to this article.

References

1 Nannoni S, de Groot R, Bell S, Markus HS. Stroke in COVID-19: a systematic review and meta-analysis. Int J Stroke. 2021;16(2):13749.

2 Perry RJ, Smith CJ, Roffe C, Simister R, Narayanamoorthi S, Marigold R, et al. Characteristics and outcomes of COVID-19 associated stroke: a UK multicentre case-control study. J Neurol Neurosurg Psychiatry. 2021; 92(3):242-8.

3 Fridman S, Bullrich MB, Jimenez-Ruiz A, Costantini P, Shah P, Just C, et al. Stroke risk, phenotypes, and death in COVID-19: systematic review and newly reported cases. Neurology. 2020;95(24):e3373-85.

4 Richter D, Krogias C, Eyding J, Bartig D, Grau A, Weber R. Comparison of stroke care parameters in acute ischemic stroke patients with and without concurrent Covid-19. A Nationwide analysis. Neurol Res Pract. 2020;2: 48. 
5 Bernard Stoecklin S, Rolland P, Silue Y, Mailles A, Campese C, Simondon A, et al. First cases of coronavirus disease 2019 (COVID-19) in France: surveillance, investigations and control measures, January 2020. Euro Surveill. 2020;25(6):2000094.

6 Nannoni S, de Groot R, Bell S, Markus HS. EXPRESS: stroke in COVID-19: a systematic review and meta-analysis. Int J Stroke. 2021; 16(2):137-49.

7 Bae S, Kim SR, Kim MN, Shim WJ, Park SM. Impact of cardiovascular disease and risk factors on fatal outcomes in patients with COVID-19 according to age: a systematic review and meta-analysis. Heart. 2021;107(5):37380.

8 Venketasubramanian N, Anderson C, Ay H, Aybek S, Brinjikji W, de Freitas GR, et al. Stroke care during the COVID-19 pandemic: international expert panel review. Cerebrovas Dis. 2021;1-17.

9 Altersberger Valerian L, Stolze Lotte J, Heldner Mirjam R, Henon H, Martinez-Majander $\mathrm{N}$, Hametner $\mathrm{C}$, et al. Maintenance of acute stroke care service during the COVID-19 pandemic lockdown. Stroke. 2021;52(5): 1693-701.

10 Calmettes J, Peres R, Goncalves B, Varlan D, Turc G, Obadia M, et al. Clinical outcome of acute ischemic strokes in patients with $\mathrm{CO}$ VID-19. Cerebrovasc Dis. 2021;1-8.

11 Fuentes B, Alonso de Leciñana M, GarcíaMadrona S, Díaz-Otero F, Aguirre C, Calleja $\mathrm{P}$, et al. Stroke acute management and outcomes during the COVID-19 outbreak: a cohort study from the madrid stroke network. Stroke. 2021;52(2):552-62.

12 Pezzini A, Grassi M, Silvestrelli G, Locatelli M, Rifino N, Beretta S, et al. Impact of SARS$\mathrm{CoV}-2$ on reperfusion therapies for acute ischemic stroke in Lombardy, Italy: the STROKOVID network. J Neurol. 2021;1-8.

13 Nguyen TN, Abdalkader M, Jovin TG, Nogueira RG, Jadhav AP, Haussen DC, et al. Mechanical thrombectomy in the era of the COVID-19 pandemic: emergency preparedness for neuroscience teams: a guidance statement from the society of vascular and inter- ventional neurology. Stroke. 2020;51(6): 1896-901.

14 Tan YK, Goh C, Leow AST, Tambyah PA, Ang A, Yap ES, et al. COVID-19 and ischemic stroke: a systematic review and meta-summary of the literature. J Thromb Thrombolysis. 2020;50(3):587-95.

15 Siow I, Lee KS, Zhang JJY, Saffari SE, Ng A, Young B. Stroke as a neurological complication of COVID-19: a systematic review and meta-analysis of incidence, outcomes and predictors. J Stroke Cerebrovas Dis. 2020; 30(3): 105549.

16 Ladopoulos T, Zand R, Shahjouei S, Chang JJ, Motte J, Charles James J, et al. COVID-19: neuroimaging features of a pandemic. J Neuroimaging. 2021;31(2):228-43.

17 Kaeuffer C, Le Hyaric C, Fabacher T, Mootien J, Dervieux B, Ruch Y, et al. Clinical characteristics and risk factors associated with severe COVID-19: prospective analysis of 1,045 hospitalised cases in North-Eastern France, March 2020. Euro Surveill. 2020;25(48): 2000895. 